\title{
Increasing Dominance With No Efficiency Effect
}

\author{
Luís M B Cabral* \\ New York University
}

August 2000

\begin{abstract}
I uncover a new force towards increasing dominance (the property whereby, in dynamic games, the leader tends to increase her lead in expected terms). The new effect results from the strategic choice of covariance in races. I assume that players must choose not the amount of resources to spend but how to allocate those resources. I show that the laggard has an incentive to chose a different path from the leader. In equilibrium, this results in the laggard choosing a less promising path, in effect trading off lower expected value for lower correlation with respect to the leader. This in turn leads to increasing dominance. In order to make the point as clear as possible and differentiate it from the forces previously characterized, I assume that no joint payoff (or efficiency) effect is present.
\end{abstract}

Keywords: Dynamic competition, increasing dominance, efficiency effect, R\&D.

JEL Code Nos.: C7, L1.

${ }^{*}$ I am grateful to Richard Gilbert, Miguel Villas-Boas, an Associate Editor and a referee for useful comments and suggestions. 


\section{Introduction}

In a dynamic $R \& D$ race, does the leader tend to get farther ahead of the rival (increasing dominance), or does the rival tend to catch up with the leader? This important question has been studied by a number of authors. Gilbert and Newbery (1982) characterize conditions under which monopoly dominance persists over time even though there are opportunities for rival firms to challenge the incumbent. Budd, Harris and Vickers (1993) identify some the basic forces leading to increasing dominance (ID) in the context of a one-dimensional dynamic model of R\&D. Cabral and Riordan (1994) provide sufficient conditions for ID is the context of dynamic competition with learning-by-doing. More recently, Athey and Schmutzler (1999) derive general conditions for increasing dominance in a reduced-form model of dynamic competition. ${ }^{1}$

One common feature of all of these models is that strategies are defined by some measure of effort in trying to move ahead of competitors. Moreover, the increasing dominance results are all based on two parts. First, the fact (which may be assumed or derived from primitive assumptions) that total payoffs are greater the greater the asymmetry between players - the efficiency effect. Second, the property of certain dynamic games that the system tends to move in the direction where joint payoff are greater. In other words: suppose that total payoffs are greater when the leader gets farther ahead in a race. Then, in equilibrium the leader will tend to get farther ahead in the race.

In this paper, I uncover an additional force towards increasing dominance, one that is based on the strategic choice of covariance. Suppose that players must choose not the amount of resources to spend but how to allocate those resources. ${ }^{2}$ Specifically, suppose that each player has a fixed amount of resources to spend and must choose between alternative paths of uncertain success. By choosing the same path, the players' success is perfectly correlated. By choosing different paths, success is independent across players. In this context, I show that the laggard in a race has an incentive to chose a different path from the leader. In equilibrium, this results in the laggard choosing a less promising path, in effect trading off lower expected value for lower correlation with respect to the leader. This in turn leads to increasing dominance.

In order to make the point as clear as possible, I assume that no joint payoff (or efficiency) effect is present. In this context, any force towards increasing dominance must originate in something other than the joint payoff (or efficiency) effect.

The paper is organized as follows. In the next section, I introduce a two-player, infinite period game where players must choose in each period between two alternative paths. Section 3 presents the main results. Section 4 includes a discussion of the results and their robustness to a number of generalizations.

\footnotetext{
${ }^{1}$ Other results related to increasing dominance include Flaherty (1980), Arthur (1989), Klepper (1996), and Bagwell, Ramey and Spuelber (1997).

${ }^{2}$ Judd (1985), Batthacharya and Mookherjee (1986), Klette and deMeza (1986), and Hoernig (1999) consider the choice of R\&D effort and/or type of effort. However, they do not address the issue of correlation across players.
} 


\section{Model definitions and assumptions}

Consider an infinite-period game with two players. In each period, the state of the game is summarized by an integer $z \in \mathbb{Z}$. Short run payoffs are summarized by the functions $p_{i}(z), i=1,2$. I assume that payoff functions are monotonic and symmetric, i.e. $p_{1}(z)$ is increasing in $z$ and $p_{2}(z)=p_{1}(-z)$ (and thus $p_{2}(z)$ is decreasing in $z$ ). By an abuse of notation that simplifies the analysis, I denote by $p(s)$ the payoff for a player who is "ahead" is state $z=s \geq 0$ (the "leader"); the payoff for the rival player (the "laggard") is therefore $p(-s)$.

One useful way of thinking about the model is that two firms attempt to move up a quality ladder (or down a cost ladder) by exerting R\&D effort. In each period, payoffs are determined by the difference in quality levels, $s=q_{i}-q_{j}$. Motion across states is therefore determined by the firms' success in moving up the ladder.

A crucial feature of the model is that players must choose between two alternative paths, $a$ and $b$; and, once a path is chosen, a fixed amount of effort is spent in following that path. If we interpret the model as one of $R \& D$ competition, then this amounts to assuming that the R\&D budget is fixed and that the only choice is between different research paths. Each path allows players to move up the ladder one step with a positive probability, $\alpha$ and $\beta$, respectively, where both $\alpha$ and $\beta$ are strictly between 0 and 1 .

If players were to choose paths based on expected value only, then the choice would be trivial $-a$ if $\alpha>\beta$ and $b$ if $\beta>\alpha$. However, selecting a particular path also implies a particular correlation with respect to the rival player's motion. Specifically, I assume that, if both players choose the same path, then either both players move up one step or neither one does. If players choose different paths, however, then the probability of success is independent across players. Finally, if players choose each of the paths with strictly positive probability, then the players' motion is positively but imperfectly correlated. Formally:

Assumption 1 Success is perfectly correlated for a given period and path, independent across periods and paths.

As an example, consider the case of two R\&D labs working on superconductors. Suppose the only choice that each lab has to make is the particular type of ceramics to use in developing a better superconductor. Since in this case R\&D is primarily a matter of finding out whether a particular material "works" or does not work, success is only a function of the material chosen by the lab, not a function of the lab itself. Assumption 1 would then follow.

A Markov strategy for player $i$ is a map $x_{i}(s)$, giving the probability of choosing path $a$ in state $s$. A pair of strategies $x_{i}(s)$, together with the (common) discount factor $\delta$, induce value functions $v_{i}(s)$. I treat value functions in terms of average period payoff, so $v_{i}(s)=(1-\delta) p_{i}(s)+\delta v_{i}^{+}$, whare $v_{i}^{+}$is player $i$ 's expected continuation value. Moreover, I restrict to symmetric equilibria. For simplicity, if with some abuse of notation, I denote strategies and value functions by $x(s)$ and $v(s)$, respectively. 
The main result of the paper is that increasing dominance results even when there is no joint payoff effect. For this purpose, I assume that total payoffs are constant, that is, independent of the state. Specifically, I assume that

Assumption $2 \quad 0 \leq p(s) \leq 1 ; p(s)+p(-s)=1$.

Assumption 2 implies that, if increasing dominance occurs, it does not result from the joint payoff effect. I also assume that a leader achieves her maximum payoff for a finite lead length:

Assumption 3 There exists an $\bar{s}$ such that, for $n>\bar{s}, p(s)=1$.

\section{Main results}

I now present the main results of the paper. Lemma 1 and Corollary 1 characterize the equilibrium when the two paths are equally promising $(\alpha=\beta)$. I show that the race then has the nature of a "matching pennies" game, the equilibrium being for players to choose each path with probability $\frac{1}{2}$. If $\alpha>\beta$, however, then the leading player chooses the most promising path with greater probability, which in turn implies ID-Proposition 1.

Lemma 1 Suppose that $\alpha=\beta$. Then, in equilibrium and for $n>\bar{s}$ it must be that

$$
x(s)=\left\{\begin{array}{cl}
1 & \text { if } x(-s)>1 / 2 \\
0 & \text { if } x(-s)<1 / 2 \\
{[0,1]} & \text { if } x(-s)=1 / 2
\end{array} \quad x(-s)=\left\{\begin{array}{cl}
0 & \text { if } x(s)>1 / 2 \\
1 & \text { if } x(s)<1 / 2 \\
{[0,1]} & \text { if } x(s)=1 / 2
\end{array}\right.\right.
$$

Proof: Expected payoff in state $s$ is given by

$$
v(s)=(1-\delta) p(s)+\delta \phi v(s-1)+\delta \phi v(s+1)+\delta(1-2 \phi) v(s),
$$

where

$$
\phi \equiv \alpha(1-\alpha)(x(s)(1-x(-s))+(1-x(s)) x(-s)) .
$$

First notice that it must be $0<v(s)<1$ for all $s$. In fact, suppose the opposite is true and that $v\left(-s^{\prime}\right)=0, v\left(-s^{\prime}+1\right)>0$. This is only possible if, in state $s^{\prime}, \phi=0$, which corresponds to the case when $x\left(s^{\prime}\right)$ and $x\left(-s^{\prime}\right)$ are equal to each other and equal to 0 or 1 . But clearly this is not an equilibrium, for in state $s^{\prime}$ the player receiving a payoff of zero would increase his or her value by choosing a different $x\left(-s^{\prime}\right)$. This argument also implies that, if $s>\bar{s}, \phi>0$.

Suppose that $\phi \neq 0$. It follows that

$$
v(s)-\frac{1}{2} v(s-1)-\frac{1}{2} v(s+1)=\frac{1-\delta}{2 \phi \delta}(p(s)-v(s)) .
$$


If $s>\bar{s}, 1=p(s)>v(s)$. Consequently, $v(s)$ is locally concave and $v(-s)=1-v(s)$ is locally convex. This implies that the leader's optimal $x(s)$ is that which minimizes $\phi$, whereas the laggard's optimal $x(-s)$ is that which maximizes $\phi$. The best responses in the lemma then follow.

In words, Lemma 1 states that the leader's best response is to "imitate" the laggard, whereas the laggard's best response is to "differentiate" from the leader. In fact, if the leader wants to maximize the probability of selecting the same path as the laggard, then she should take path $a$ with probability 1 if the laggard selects $a$ with probability greater than $1 / 2$, as indicated by the Lemma. Likewise, if the laggard wants to minimize the probability of selecting the same path as the leader, then he should take path $b$ if the leader selects $a$ with probability greater than $1 / 2$, as indicated by the Lemma.

The intuition for this result is that a leader's current payoff is greater than her discounted value, that is, "things can only get worse." To be more precise: the leader is worse off in equilibrium than she would be if the state were never to change. Moreover, in equilibrium the system moves to left and to the right with probability $1 / 2$ each. These facts imply that the leader's value function is concave: she has less to gain from extending her lead than she has to lose from being caught up by the laggard. She thus prefers to minimize the variance of motion across states, which she does by maximizing the correlation with respect to the laggard. Conversely, the laggard's current payoff is lower than his discounted value, that is, "things can only get better." This implies, by a similar argument, that his value function is convex: he has less to lose from letting his lag extend than he has to gain from catching up with the leader. He thus prefers to maximize the variance of motion across states, which he does by minimizing the correlation with respect to the leader.

An immediate implication of Lemma 1 is that, as mentioned above, the game has the nature of a "matching pennies" game, the equilibrium of which is for players to equally mix between the two possible paths:

Corollary 1 Suppose that $\alpha=\beta$. Then, in equilibrium and for $s>\bar{s}, x(s)=x(-s)=1 / 2$.

Lemma 1 and Corollary 1 characterize the equilibrium when the two paths are equally promising $(\alpha=\beta)$. I now consider the case when one of the paths is more promising $(\alpha>\beta)$ and show that increasing dominance results in equilibrium:

Proposition 1 (increasing dominance) There exists an $\epsilon$ such that, if $\beta<\alpha<\beta+\epsilon$, then in equilibrium and at state $s>\bar{s}$ the gap between leader and follower increases in expected value.

Proof: From the proof of Lemma 1, we conclude that, in equilibrium, players mix between the two paths. In fact, value functions are strictly concave (leader) and convex (laggard), so that, even if $\alpha$ is changed by a small amount, the nature of the best response functions remains as before: the leader preferring to "imitate" the laggard, the latter preferring to differentiate from the former. 
Expected payoff at state $s$ is given by

$$
v(s)=(1-\delta) p(s)+\delta \phi^{-} v(s-1)+\delta \phi^{\circ} v(s)+\delta \phi^{+} v(s+1),
$$

where

$$
\begin{aligned}
\phi^{-} & \equiv x(s)(1-x(-s))(1-\alpha) \beta+(1-x(s)) x(-s)(1-\beta) \alpha \\
\phi^{+} & \equiv x(s)(1-x(-s)) \alpha(1-\beta)+(1-x(s)) x(-s) \beta(1-\alpha) . \\
\phi^{\circ} & \equiv 1-\phi^{-}-\phi^{+}
\end{aligned}
$$

The fact that the leader mixes implies that the right-hand side of the value function is invariant with respect to $x(s)$. Substituting 1 and 0 for $x(s)$, equating, and solving for $x(-s)$, we get

$$
x(-s)=\frac{(\alpha+\beta-2 \alpha \beta) v(s)-\beta(1-\alpha) v(s-1)-\alpha(1-\beta) v(s+1)}{(\alpha+\beta-2 \alpha \beta)(2 v(s)-v(s-1)-v(s+1))} .
$$

Differentiating with respect to $\alpha$ at $\alpha=\beta$ yields

$$
\left.\frac{\partial x(-s)}{\partial \alpha}\right|_{\alpha=\beta}=-\frac{v(s+1)-v(s-1)}{4 \alpha(1-\alpha)(2 v(s)-v(s-1)-v(s+1))} .
$$

Since $p(s) \leq 1, n<\bar{s}$ (strict inequality for $s<-\bar{s}$ ) and $p(s)=1, s>\bar{s}, v(s)$ is increasing in $s$ for $s>\bar{s}$. Moreover, by the same argument as in the proof of Lemma 1,v $v(s)$ is concave for $s>\bar{s}$. It follows that the above derivative is negative, which in turn implies that $x(-s)<1 / 2$ (recall that, for $\alpha=\beta, x(s)=x(-s)=1 / 2$ ). An analogous argument implies that $x(s)>1 / 2$. Since path $a$ is better than $b$ (in expected value), the result follows.

The above results are based on several assumptions regarding the value of $\alpha$ and $s$. The results are tight in the sense that one can find counterexamples when those assumptions fail. Specifically, if $\alpha$ is much greater than $\beta$, it is no longer the case that players mix between the two paths. In fact, for $\alpha$ sufficiently greater than $\beta$, both players choose path $a$. Moreover, if $s$ is less than $\bar{s}$, one can find examples whereby the system moves toward zero in expected value: it suffices to assume that $\delta$ is close to zero and $p(s)$ convex.

Alternative formulations of main result. The previous results are limited in that they only apply for the case when the leader is "far" ahead of the laggard (that is, for $s$ large enough). However, imposing additional restrictions on the value of $\delta$, I can prove similar versions of the increasing dominance result which apply at every state. The following results dispense with Assumption 3.

Proposition 2 Suppose that $p(s)$ is strictly concave for $n>0$. There exist $\epsilon, \bar{\delta}>0$ such that, if $\beta<\alpha<\beta+\epsilon$, and $\delta<\bar{\delta}$, then the gap between leader and follower increases in expected value. 
Proof: If $\delta$ is close to zero, then the payoff function provides a first-order approximation to the value function. Concavity of $p(s)$ therefore implies concavity of $v(s)$. The rest of the proof proceeds as as in Lemma 1.

Proposition 3 Suppose that $p(s)>\frac{1}{2}$ iff $n>0$. There exist $\epsilon, \bar{\delta}>0$ such that, if $\beta<\alpha<$ $\beta+\epsilon$, and $\delta>\bar{\delta}$, then the gap between leader and follower increases in expected value.

Proof: Recall that $v(s)=(1-\delta) p(s)+\delta\left(\phi^{-} v(n-1)+\phi^{\circ} v(s)+\phi^{+} v(n+1)\right)$. Together with $v(s)+v(-s)=1$, this implies that $\lim _{\delta \rightarrow 1} v(s)=\frac{1}{2}$. Since $p(s)>\frac{1}{2}$ iff $n>0$, it follows that for $\delta$ large enough, $p(s)>v(s)$. The proof then proceeds as in Lemma 1 .

\section{Discussion}

Although my model implies increasing dominance, the reasons for the result are in stark contrast to standard increasing dominance results. In the latter, total payoffs are increasing when increasing dominance takes place. Typically, this results from a convex payoff function, that is, a function with the properties that the leader has more to gain from extending her lead than the laggard has to lose from falling farther behind. By contrast, my model features constant total payoffs, so that the above effect is absent. Instead, the crucial feature of the equilibrium is that the leader has less to gain from moving farther ahead than she has to lose from being caught up by the laggard, whereas the laggard has more to gain from moving closer to the leader than he has to lose from falling farther behind. This implies that the leader prefers low variance of motion in the state space, or, equivalently, high correlation with respect to the laggard; whereas the laggard prefers the opposite, that is, low correlation with respect to the leader.

In the standard increasing dominance results, convexity of the payoff function translates into equilibrium strategies whereby the leader makes a greater effort than the laggard. By contrast, my model features constant total effort, so that the previous effect is absent. Instead, convexity of the laggard's value function translates into an equilibrium strategy whereby the laggard trades off a lower expected value for a lower correlation with respect to the leader.

The results in the previous sections are based on a strong set of assumptions. These assumptions are in some cases necessary. In other cases, however, they are only made for simplicity and could be generalized. In what follows, I discuss the importance of each of the assumptions.

The assumption that there are only two possible $R \& D$ paths is not necessary. The result can be generalized to the case when firms have $L$ available paths, whereby choosing $l_{i}$ implies moving up one step with probability $\lambda_{i}$. When $\lambda_{i}=\bar{\lambda}, \forall i$, the equilibrium is for both firms 
to choose each path $i$ with probability $1 / L$. Moreover, if $\lambda_{i}>\lambda_{i+i}$ and $\lambda_{i} \in[\bar{\lambda}-\epsilon, \bar{\lambda}+\epsilon]$, then, for $\lambda_{i}>\lambda$, the leader follows path $i$ with probability greater than $1 / L$ and the laggard with probability lower than $1 / L$. This in turn results in increasing dominance.

The assumptions that (a) the motion technologies only allow for one-step moves and (b) total payoff is constant are important for the proof of Propositions 1-3. In Cabral (1999), I consider the case of more complex motion technologies and payoff functions. I am able to (conditionally) characterize the Nash equilibrium but unable to prove existence or uniqueness of equilibria.

The assumption that the outcome of $R \& D$ is perfectly correlated across players when they follow the same path is made for simplicity and not at all crucial. What matters is that (1) correlation is greater when players follow the same path than when they follow separate paths; and (2) correlation is the same for each path. ${ }^{3}$

I have assumed that the set of available paths is common knowledge and that choices are always simultaneous (that is, each player does not observe the other player's choice until making his or her own choice). Departing from these assumptions would lead to a very different game structure; it is not clear whether ID would still hold. ${ }^{4}$

Finally, the assumption that there are only two players is quite important. In competitive sailing - a sport that, in many respects, is similar to the game in this paper - there is also a crucial difference between two boats (match racing) and more than two boats (normal racing). In match racing, the optimal strategy for the leading boat is quite clear: to cover the laggard (which implies a high level of correlation). In normal racing, by contrast, the optimal strategy is significantly more complicated. I suspect the same would be true in R\&D races with more than two players.

\footnotetext{
${ }^{3}$ In particular, it cannot be the case that correlation is greater when both players follow path $b$ than when both players follow path $a$. If that were the case, the opposite of ID might take place.

${ }^{4}$ I conjecture that, if the leader (respectively, the laggard) gets to observe its rival's choice before making its own choice, then this implies an added effect towards ID (respectively, a counterveiling effect).
} 


\section{References}

Arthur, W Brian (1989), "Competing Technologies, Increasing Returns, and Lock-In by Historical Events," The Economic Journal 99, 116-131.

Athey, Susan, And Armin Schmutzler (1999), "Innovation and the Emergence of Market Dominance," MIT and Universität Zürich, August.

Bagwell, Kyle, Garey Ramey, and Daniel F Spuelber (1997), "Dynamic Retail Price and Investment Competition," Rand Journal of Economics 28, 207-227.

Bhattacharya, Sudipto, And Dilip Mookherjee (1986), "Portfolio Choice in Research and Development," Rand Journal of Economics 17, 594-605.

Budd, Christopher, Christopher Harris, And John Vickers (1993), "A Model of the Evolution of Duopoly: Does the Asymmetry Between Firms Tend to Increase or Decrease?," Review of Economic Studies 60, 543-574.

Cabral, Luís M B (1999), "Football, Sailing, and R\&D: Dynamic Competition With Strategic Choice of Variance and Covariance," http://www.stern.nyu.edu/ lcabral/.

Cabral, Luís M B, And Michael H Riordan (1994), "The Learning Curve, Market Dominance and Predatory Pricing," Econometrica 62, 1115-1140.

Flaherty, M Therese (1980), "Industry Structure and Cost-Reducing Investment," Econometrica 48, 1187-1209.

Gilbert, Richard J, and David M G Newbery (1982), "Preemptive Patenting and the Persistence of Monopoly Power," American Economic Review 72, 514-526.

Hoernig, Steffen (1999), "A Model of Dynamic R\&D Competition With Endogenous Innovation Targets and Leapfrogging," Universidade Católica Portuguesa, February.

Klepper, Steven (1996), "Entry, Exit, Growth, and Innovation over the Product Life Cycle," American Economic Review 86, 562-583.

Klette, Tor Jakob, And David de Meza (1986), "Is the Market Biased Against Risky R\&D?," Rand Journal of Economics 17, 133-139. 